\title{
On the nature of moving magnetic feature pairs around sunspots
}

\author{
Jun Zhang ${ }^{1,2}$, S. K. Solanki ${ }^{1}$, and Jingxiu Wang ${ }^{2}$ \\ 1 Max-Planck-Institut für Aeronomie, 37191, Katlenburg-Lindau, Germany \\ e-mail: hangj@linmpi.mpg.de; solanki@linmpi.mpg.de \\ 2 National Astronomical Observatories, Chinese Academy of Sciences, Beijing 100012, China \\ e-mail:wjx@ourstar.bao.ac.cn;zjun@ourstar.bao.ac.cn
}

Received 25 September 2002 / Accepted 3 December 2002

\begin{abstract}
Employing data recorded by the Michelson Doppler Imager (MDI) instrument on the Solar and Heliospheric Observatory (SOHO), we have identified 144 pairs of opposite magnetic polarity moving magnetic features (MMFs) in two active regions (NOAA ARs 8375 and 9236). The following results are obtained: (1) The majority of MMF pairs first appears at a distance of 1000 to $5000 \mathrm{~km}$ from the outer boundary of the sunspot, although MMF pairs appearing closer to the sunspot may be missed. (2) MMF bipoles are not randomly oriented. The member of an MMF pair further from the sunspot has the polarity of the parent sunspot in $85 \%$ of the cases. Furthermore, the orientations of MMF pairs are associated with the twist of the sunspot superpenumbra deduced from $\mathrm{H} \alpha$ images. (3) The mean lifetime of the studied MMFs is around 4 hours. (4) The separation between the two polarities of the MMFs falls in the range of 1100-1700 km. This separation remains almost unchanged, even decreases slightly as the MMF pairs move outwards. (5) MMFs are observed to cluster at particular azimuths around the parent sunspot, in particular in AR 8375. (6) MMF pairs move approximately radially outward from sunspots at an average speed of around $0.5 \mathrm{~km} \mathrm{~s}^{-1}$. Their motion is deflected towards large concentrations of magnetic flux of opposite polarity to that of the parent sunspot. A qualitative model based on these and other observations is presented. MMF pairs are proposed to be part of a U-loop emanating from the sunspot's magnetic canopy. Possible mechanisms leading to the formation of such a loop are discussed.
\end{abstract}

Key words. Sun: chromosphere - Sun: magnetic fields - sunspots

\section{Introduction}

Moving magnetic features (MMFs) are small magnetic features that move away from a sunspot to the periphery of the surrounding moat (Vrabec 1971; Harvey \& Harvey 1973; Muller \& Mena 1987; Brickhouse \& LaBonta 1988; Lee 1992). The MMFs are generally thought to be transported by the outward moat flow. There are two kinds of MMFs: unipolar and mixed polarity (Harvey \& Harvey 1973; Ryutova et al. 1998; Yurchyshyn et al. 2001). Shine \& Title (2001) have actually proposed three types, by distinguishing between unipolar MMFs with the same and those with the opposite polarity to that of the parent sunspot. Harvey \& Harvey (1973) proposed a model in which magnetic flux is removed from the sunspot at the photospheric level. They show a figure in which a flux tube breaking away from the sunspot forms a sea serpent. MMFs are then the intersections of this flux tube with the solar surface. An alternative possibility was suggested by Wilson (1973, cf. Spruit et al. 1987). In his model, a thin magnetic flux tube is detached from the main flux of the sunspot well below the

Send offprint requests to: S. K. Solanki

e-mail: solanki@linmpi.mpg.de surface. The detached tube floats turbulently to the surface, developing twists and kinks, which are seen as MMFs once it reaches the solar surface. In this case too, a structure similar to a sea serpent can be formed. Yet another proposal is due to Wilson (1986). He proposes that the two MMFs forming a pair are connected by an O-loop. Ryutova et al. (1998) have modelled MMF pairs as $\Omega$ loops emerging from below. They propose that these loops are kinks in a horizontal flux tube lying below the solar surface. They model the propagating kinks as a solitary wave. Thomas et al. (2002) propose that the horizontal flux tubes are kept buried through magnetic flux pumping.

Recently, Yurchyshyn et al. (2001) studied 28 MMF pairs, using Big Bear Solar Observatory (BBSO) observations of the longitudinal magnetic fields of two large sunspots. They find that MMFs are not randomly oriented. The magnetic elements having the same polarity as the sunspot are located further from the sunspot (which contradicts Shine \& Title 2002). Furthermore, they find a correlation between the orientation of the MMF bipoles and the twist of the sunspot superpenumbra, as deduced from $\mathrm{H} \alpha$ images. To us this suggests that the MMFs are still attached to the sunspot's superpenumbral canopy magnetic field. This idea is further supported by the observation 
of Harvey \& Harvey (1973) that the $\mathrm{H} \alpha$ fibrils in the superpenumbra are aligned precisely with the direction of motion of the MMFs.

In this paper we study a larger sample of MMFs than Yurchyshyn et al. (2001) to test whether their results are statistically significant (or if Shine \& Title 2001 are correct regarding the dipole orientation) and to further constrain properties of MMFs (e.g. we follow all the studied MMFs from birth to death). We also present a new model of MMFs aimed at reproducing the newer data.

\section{Observations and analysis}

We use magnetic field observations carried out by the Michelson Doppler Imager, MDI (Scherrer et al. 1995) on the olar and Heliospheric Observatory (SOHO). MDI was employed in the high-resolution mode $(0.625$ arcsec per CCD pixel and a 1 min cadence). Observations of two active regions, AR 8375 and AR 9236, are analyzed. We identified 93 MMF pairs from the 40 hours (from 1998 November 3 18:53 UT to November 5 10:51 UT) of magnetograms of AR 8375, and 51 MMF pairs from the 48 hour (from the beginning of 2000 November 23 to the end of November 24) sequence of magnetograms of AR 9236. During the observations, the two active regions were located near the central meridian (AR 8375: N18 W06; AR 9236: N23 W05). Note that AR 8375 is one of the active regions studied by Yurchyshyn et al. (2001), but we consider a longer time series, which allows us to investigate a larger number of MMFs. We also consider additional parameters of the MMFs. The two active regions have a similar magnetic structure: a relatively large compact leading sunspot of positive polarity and an extended negative polarity region. At the time of observation both active regions still exhibited some flux emergence. The studied MMFs emerged successively from and around the leading sunspots.

Here we concentrate on MMF pairs. Each MMF pair was reliably identified on tens of successive magnetograms. We selected only well-isolated MMF pairs. This may bias our selection towards tighter pairs, but reduces the risk of wrongly assigning two unconnected MMFs to each other. Figure 1 shows BBSO H $\alpha$ images (left) of AR 8375 and AR 9236, respectively, corresponding MDI line-of-sight magnetograms (middle), as well as corresponding MDI continuum images (right). The dotted curves in the continuum images outline the boundary of the penumbra. The $\mathrm{H} \alpha$ filaments around the sunspot in AR 8375 are twisted counterclockwise, while the filaments in AR 9236 are twisted clockwise.

For each pair of MMFs we determined a set of parameters. These include the total magnetic flux of the pair, the net magnetic flux, the separation between the two polarities, the azimuthal angle $\beta$ relative to the centre of the sunspot, the orientation $\alpha$ of the MMF pair (i.e. of the line connecting the two polarities) relative to the radial direction measured from the centre-of-gravity of the sunspot, the lifetime of magnetic elements of MMF pairs, the speed and direction of propagation, the distance from the edge of the penumbra at which the MMF was first seen, etc. The method used to study orientation of MMF pairs was similar to that of Yurchyshyn et al. (2001).

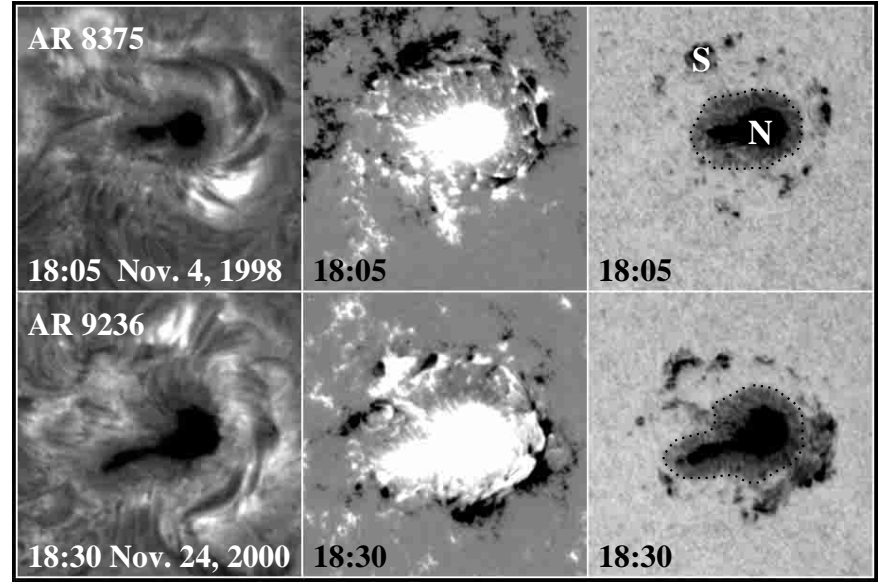

Fig. 1. Left: chromospheric $\mathrm{H} \alpha$ images of AR NOAA 8375 (top) and NOAA 9236 (bottom) taken at BBSO; middle: corresponding MDI longitudinal magnetograms; right: corresponding MDI continuum images. The field-of-view of all images is $172^{\prime \prime} \times 172^{\prime \prime}$.

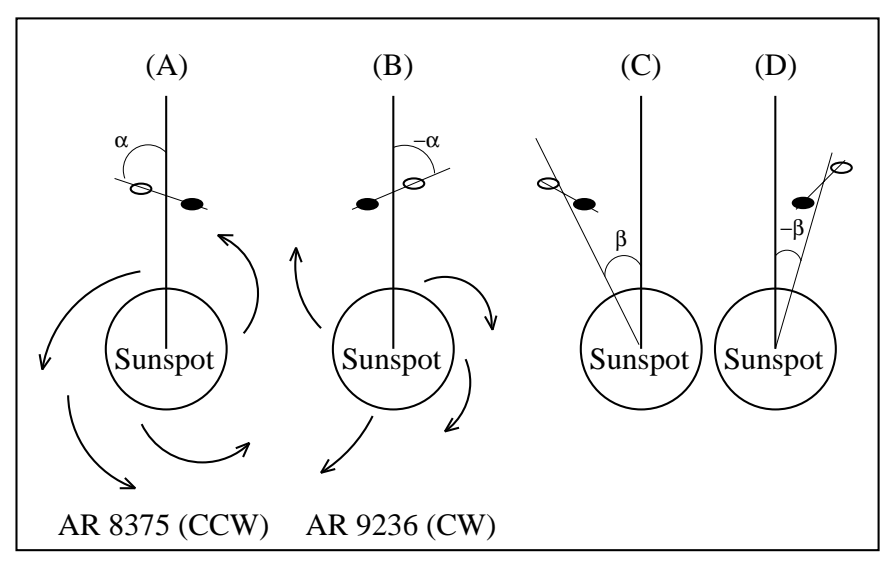

Fig. 2. Definition of angles $\alpha$ and $\beta$.

Figure 2 illustrates the definition of angles $\alpha$ and $\beta$. Angle $\alpha$ represents the angle of the axis of an MMF bipole with respect to the radial direction from the sunspot center. It is positive in the counter-clockwise direction. Angle $\beta$ is defined as the angle between the line connecting the center-of-gravity of an MMF pair with the sunspot center and the line from sunspot center to the north. $\beta$ increases in the counter-clockwise direction from 0 to $180^{\circ}$; in clockwise direction, $\beta$ runs from 0 to $-180^{\circ}$.

\section{Properties of moving magnetic features}

In this study, the long time series of magnetograms of high temporal and spatial resolution enable us to identify a large sample of MMF pairs, and to study their evolution. We identify 144 MMF pairs, 93 of them in AR 8375, the remainder, in AR 9236. All pores, i.e. features appearing dark in the continuum, were excluded from our sample. Furthermore, in AR 9236 we do not consider any features between $\beta$ values of approximately $-90^{\circ}$ and $-180^{\circ}$ in order to avoid confusion with the other flux bordering on the sunspot there. 


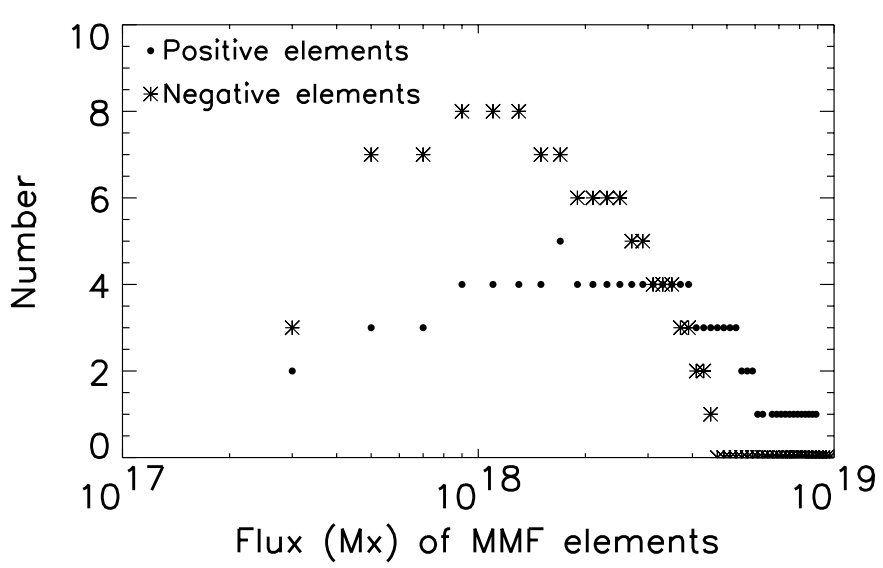

Fig. 3. Flux distributions of the positive and negative polarity elements of 144 MMF pairs.

\subsection{Magnetic flux}

A histogram of the flux of individual MMF elements is plotted in Fig. 3 separately for elements of both polarities. The mean flux per MMF element is found to be $3.6 \times 10^{18} \mathrm{Mx}$. This agrees well with the average value of $5 \times 10^{18} \mathrm{Mx}$ deduced from Advanced Stokes polarimeter data (Martínez Pillet 2002). The distributions for the two polarities differ systematically, with positive polarity elements being on the whole larger, so that we expect from Fig. 3 a net positive polarity flux to be carried away from the two investigated (positive polarity) sunspots. Integration over the histogram confirms this.

In AR 8375, the total, unsigned flux carried by the $93 \mathrm{MMF}$ pairs analyzed by us is $6.8 \times 10^{20} \mathrm{Mx}$, with a net flux of $1.2 \times 10^{20} \mathrm{Mx}$. In AR 9236 about $4.2 \times 10^{20} \mathrm{Mx}$ appears in the form of MMF pairs, while the net flux is $1.4 \times 10^{20} \mathrm{Mx}$. We also find that in both active regions the net flux of MMF pairs has the same polarity as the parent sunspots. Unfortunately, we cannot compare the net flux carried by the MMFs with a possible flux loss by the parent sunspots, since the MDI field strength and flux measurements are unreliable in sunspot umbrae (R. Bush, private communication). In addition, the above numbers only reflect the flux carried by MMF pairs, to which the flux in MMFs which we could not unambiguously assign to a pair needs to be added in order to obtain the total and net flux carried by all MMFs away from the sunspot. Also, the flux measurements of the MMFs are of limited accuracy. Systematic differences between the flux carried by the two polarities can arise if, for example, the field of the following and leading MMF of a pair are not equally inclined to the vertical.

\subsection{Location of first appearance}

The upper frame of Fig. 4 shows the distribution of the distance to the sunspot penumbral boundary at which the MMF pairs first appeared. Negative values of the distance mean that the corresponding MMF pairs appeared inside the penumbra (i.e. inside the two closed dotted curves in the continuum images in Fig. 1). Seven of the total of $144 \mathrm{MMF}$ pairs were first seen within the penumbra, although we cannot rule out that more were missed against the relatively strong penumbral
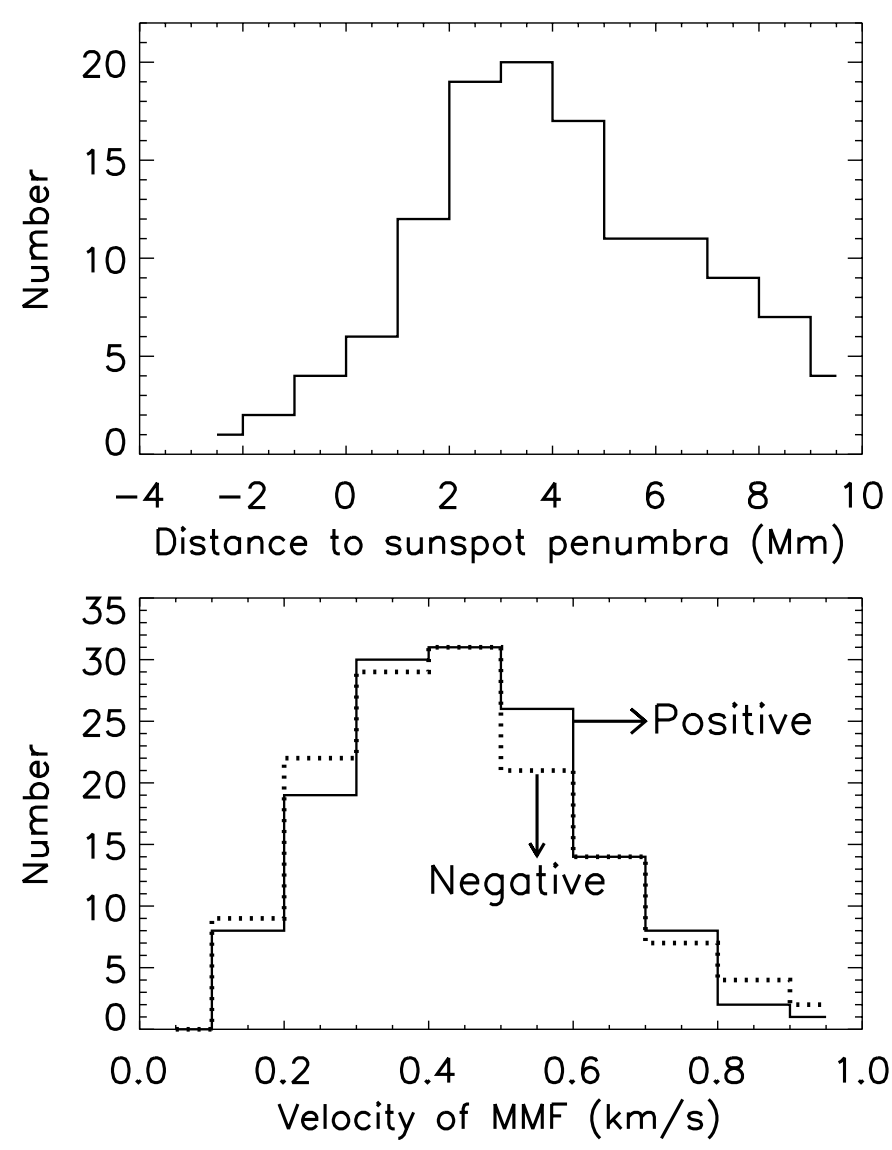

Fig. 4. Upper frame: histogram of the distance of first appearance of MMF pairs from the sunspot boundary; lower frame: histogram of the proper motion speeds of the MMFs.

signal. The mean distance at first appearance is $4500 \mathrm{~km}$, with the peak of the distance distribution lying in the range 1000$5000 \mathrm{~km}$. In other words, the majority of MMF pairs first appears at 2-7 arcsec from the penumbral boundary. Note that this distance is measured from the centre-of-gravity of the MMF, so that the polarity nearer to the sunspot started 500-1000 km closer to the penumbral boundary (see Sect. 3.5).

\subsection{Motion}

The lower frame of Fig. 4 shows the distribution of the proper motion velocity of 288 elements belonging to 144 MMF pairs, with the dotted line referring to negative MMF elements, the solid line to positive elements. The velocity ranges from 0.1 to $0.95 \mathrm{~km} \mathrm{~s}^{-1}$, with the peaks of both distributions lying near $0.4 \mathrm{~km} \mathrm{~s}^{-1}$. The average velocity is $0.45 \mathrm{~km} \mathrm{~s}^{-1}$ (irrespective of the polarity), which is lower than that found by Harvey \& Harvey (1973). Note that we measure the speed averaged over the full lifetime of each MMF element. Often the speed changes as a function of time. We have noticed that at least some MMFs initially move quite fast, but later slow down to the typical speed of supergranular outflow or moat flow. This may be responsible for the discrepancy to the results of Harvey \& Harvey (1973). A systematic study of this effect sill needs to be carried out, however. 


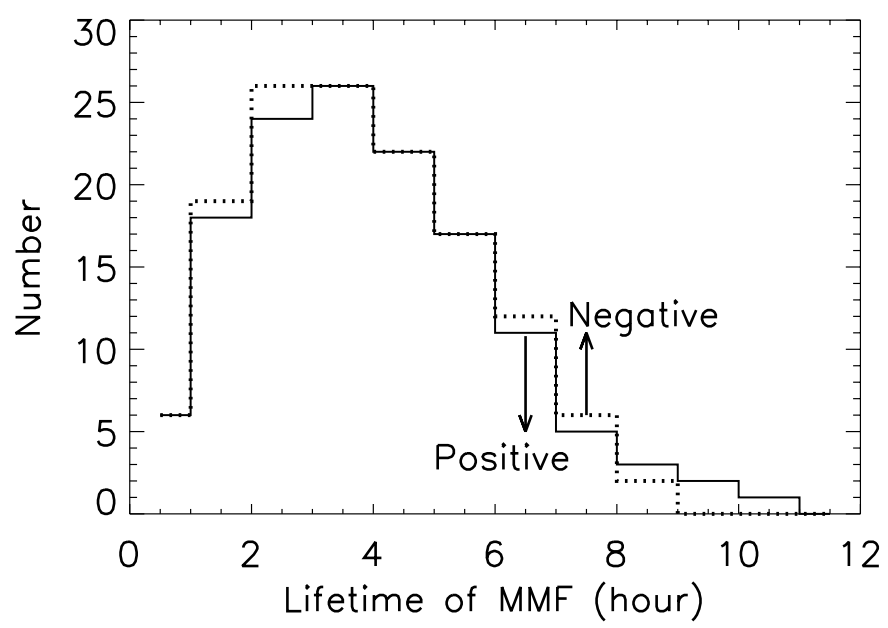

Fig. 5. Histogram of MMF lifetimes.

\subsection{Lifetime}

We have followed MMF pairs from birth to death and have determined their lifetimes. The birth of an element of an MMF pair is defined as the time when it appears as a readily recognizable entity above the background signal or the noise level of approximately $20 \mathrm{G}$ in the magnetograms. An element of an MMF pair dies for our purposes when it either diffuses into weak fields below the noise level, cancels with an element of opposite polarity or merges with field of the same polarity. In most of the studied cases, the elements of MMF pairs die by decaying. Figure 5 shows the lifetime distribution of all 288 elements of the studied MMF pairs, with the dotted line referring to MMF elements of negative polarity, the solid line to positive elements. The lifetime ranges from 0.25 to 11.0 hours, with the peak of the distribution lying close to 3.5 hours. The average lifetime is 3.9 hours.

\subsection{Separation between the two members of an MMF pair}

Another interesting parameter of MMF pairs is the separation between the opposite polarity elements. The upper frame of Fig. 6 displays the histogram of the separation both shortly after the MMFs are born (solid curve) and shortly before they disappear (dashed curve). The mean separation between the two elements of an MMF pair is about $1500 \mathrm{~km}$, shortly after birth, while at the end it is $1380 \mathrm{~km}$. Thus the separation tends to decrease slightly, by $120 \mathrm{~km}$ on average, over the lifetime of an MMF, as shown in the lower frame of Fig. 6. However, it is significant, that the average separation does not increase by more than $20 \%$ over the whole lifetime of an MMF pair. This is remarkably stable compared with the behaviour of freshly emerged $\Omega$ loops (e.g. in ephemeral regions). Our result contradicts the report by Shine \& Title (2001) that the two polarities separate with time at a rate of $100 \mathrm{~m} \mathrm{~s}^{-1}$. Over the mean lifetime of an MMF this would lead to an expansion of $1400 \mathrm{~km}$, which was shown by none of the MMFs in our sample. We cannot rule out that the application of other criteria to the identification of MMF pairs (i.e. which two MMFs belong together)
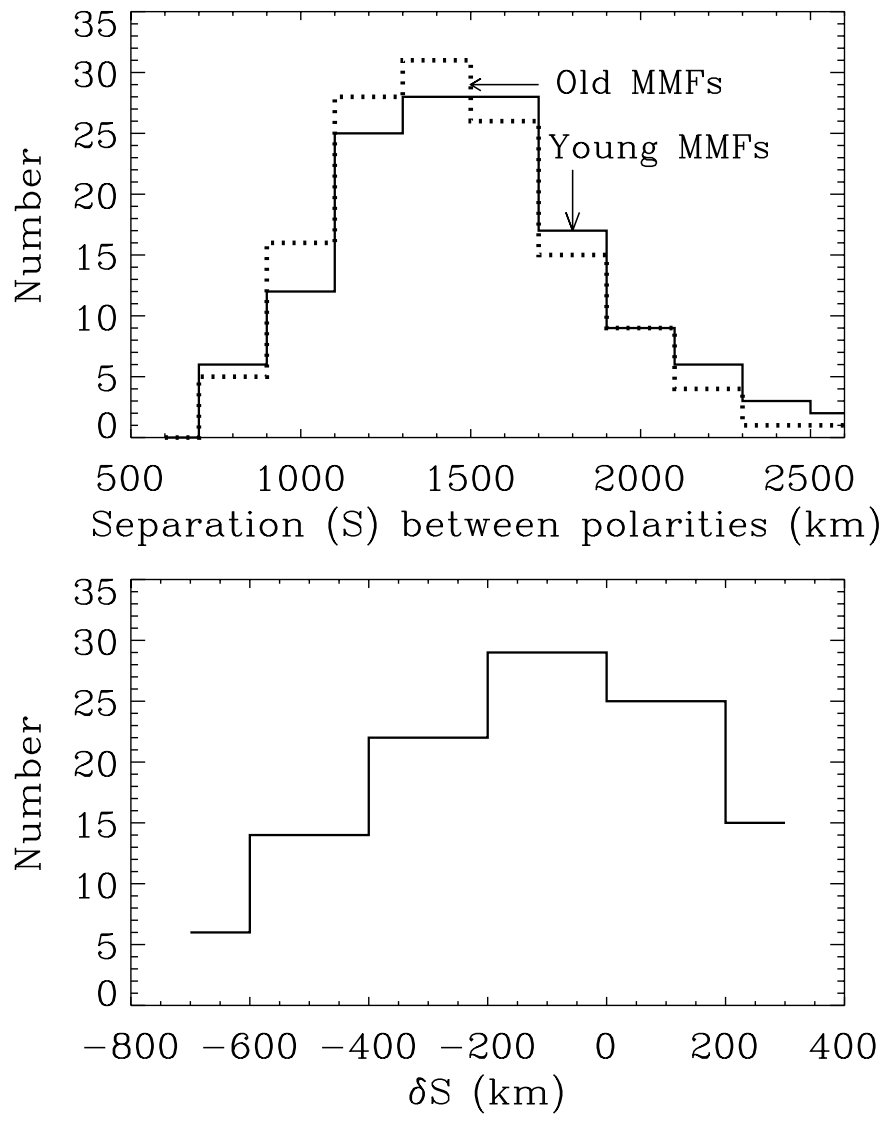

Fig. 6. Upper frame: histogram showing the separation between the individual MMFs forming a pair; lower frame: histogram showing the difference between this separation near the end and near the beginning of the life of an MMF pair.

could lead to other results. In our case we picked pairs on the basis of a relatively small distance between the two polarities.

\subsection{Orientation of MMF bipoles}

A central property needed to decide the nature of MMFs is the orientation of MMF bipoles. Based on our current sample of $144 \mathrm{MMF}$ pairs, we find that the member of an MMF pair with the polarity of the parent sunspot is further away from the sunspot in $85 \%( \pm 12 \%)$ of the cases in AR 8375 and in $87 \%( \pm 7 \%)$ in AR 9236 . This result supports the finding of Yurchyshyn et al. (2001), who studied 28 MMF pairs.

Another quantity studied by Yurchyshyn et al. (2001) was the orientation of the axes of the MMF pairs relative to the radial direction from sunspot centre. We describe this orientation by the angle $\alpha$ (Fig. 2). The upper panel of Fig. 7 shows the distribution of $\alpha$. For AR 8375, only 18 of $93 \alpha$ values are negative and the mean $\alpha$ is $38^{\circ}$. For AR 9236, on the contrary, only 12 of $51 \alpha$ are positive and the mean $\alpha$ is $-25^{\circ}$. The orientation of the MMFs is thus consistent with the orientation of the superpenumbral $\mathrm{H} \alpha$ fibrils (compare with Fig. 1). Hence the orientation found by us is completely in agreement with the results of Yurchyshyn et al. (2001), but does not agree with the statement made by, e.g., Shine \& Title (2001) that the MMF with the sunspot's polarity lies closer to the sunspot. Note the 

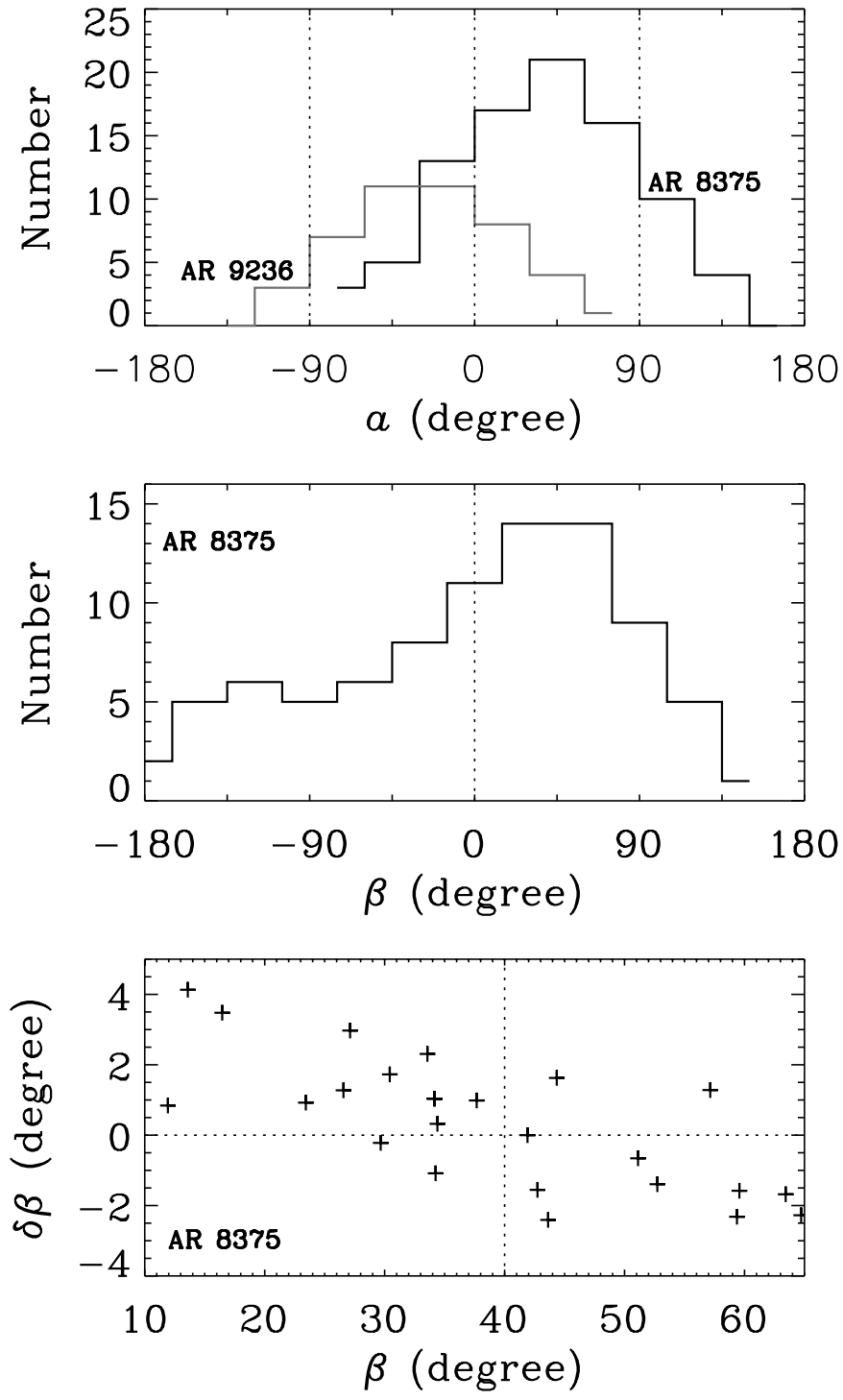

Fig. 7. Upper frame: histogram of measurements of the angle $\alpha$ in the two investigated active regions; middle frame: histogram of the number of MMFs for a given angle $\beta$ in AR NOAA 8375; lower frame: the relationship between $\beta$ and the change of $\beta$ over an MMF's lifetime. Only a restricted $\beta$-range is considered (see text for details).

considerable width of the distribution of $\alpha$ values (Fig. 7). It is therefore not surprising that Lee (1992), who only considered 5 MMF pairs, concluded that they are randomly oriented (although a look at his Fig. 1 does reveal that, within a given MMF pair, the MMF with the polarity of the sunspot tends to lie further away).

One surprising result is that for AR 8375 the number of MMF pairs is not evenly distributed all around the sunspot. Instead they are most common at azimuths $\beta$ between 0 and $90^{\circ}$, with a peak in the distribution at $\beta \approx 40^{\circ}$, while hardly any were seen near $180^{\circ}$ (middle panel of Fig. 7). Comparing to Fig. 1 we notice that the line with $\beta$ equals $40^{\circ}$ connects the main N-polarity spot with a smaller S-polarity spot (see the continuum image in Fig. 1 of AR 8375). Hence the largest number of MMFs are found in the region between the two opposite polarity spots, travelling from the larger spot towards
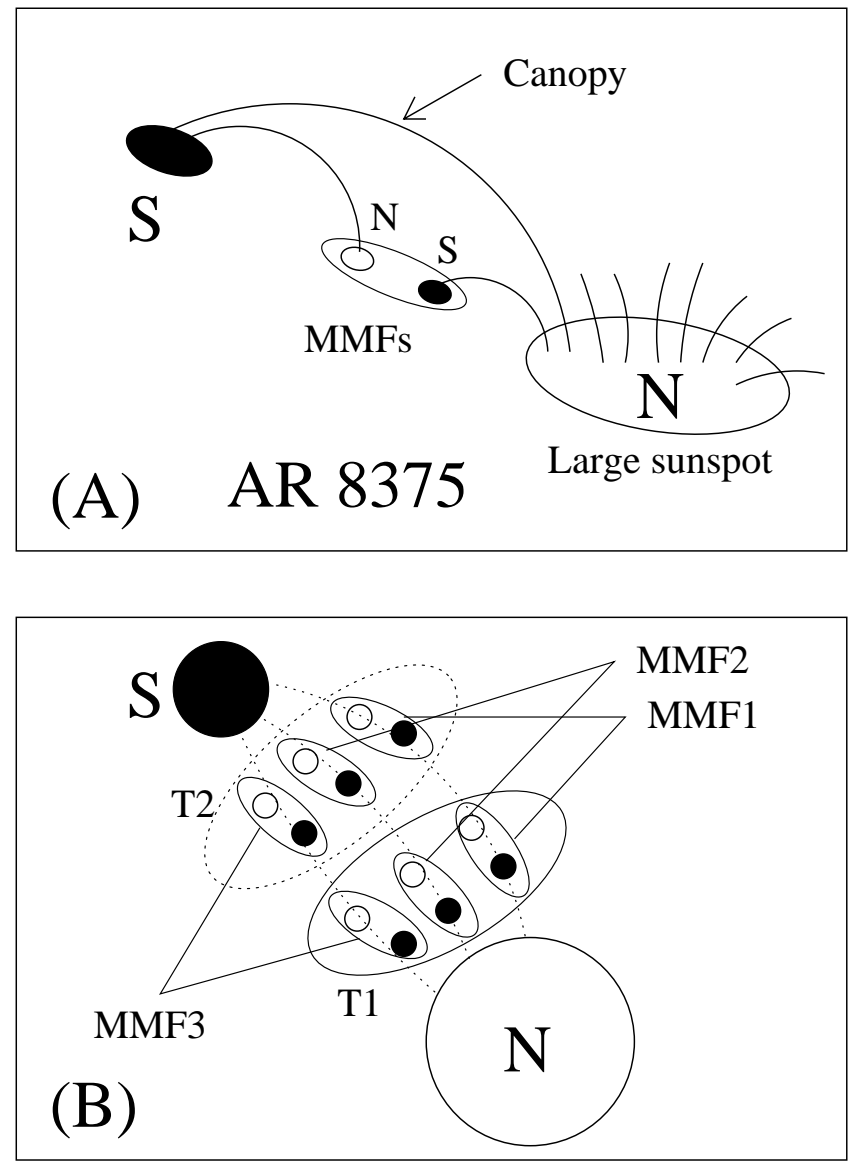

Fig. 8. Sketch of the geometry (upper frame) and motion (lower frame) of MMF pairs in AR 8375 between the main spot (marked $\mathrm{N}$ ) and the smaller opposite polarity spot (marked S).

the smaller. Some of the MMFs disappear before reaching the smaller spot, others merge or cancel with it, depending on their polarity. We also noticed that the MMFs lying between $\beta=10^{\circ}$ and $65^{\circ}$ follow trajectories which are focussed towards the smaller sunspot. This is shown in the lowest panel of Fig. 7, in which the difference between $\beta$ at the end and beginning of an MMF's lifetime is plotted versus its initial $\beta$. For MMF pairs with initially $10^{\circ}<\beta<40^{\circ}$, the $\beta$ difference is positive, while for MMF pairs with $40^{\circ}<\beta<65^{\circ}$, the difference is negative. For AR 9236 the distribution of the number of MMF pairs as a function of $\beta$ also shows a peak in the direction of the largest nearby patch of opposite polarity field (at around $\beta=50^{\circ}$ ), although it is not as pronounced as for AR 8375. This concentration is sketched in the lower frame of Fig. 8. Support for this conclusion comes from a horizontal flow map produced by Yurchyshyn \& Wang (2001). Furthermore, Yurchyshyn (private communication) points out that pairs of MMFs are often clustered. It is unclear to us, however, whether such a clustering depends on details of the magnetic and velocity fields or is a basic property of MMFs.

\section{A qualitative model}

The results of Sect. 3 and of Yurchyshyn et al. (2001) are best explained if the MMF pairs remain attached to the parent 
sunspot throughout their life. We propose that MMF pairs are formed when the field lines in a small part of the magnetic canopy dip down to produce a U-loop. This is qualitatively similar to the picture of the magnetic structure of what Bernasconi et al. (2002) call Moving Dipolar Features seen to move towards a forming sunspot.

How is such a U-loop formed? There are various possibilities. One is that it is driven by an instability caused by the presence of a shear flow at the base of the canopy. Such an instability has been studied for a flux tube carrying a flow by Holzwarth \& Schüssler (2002). An instability, possibly of this type, that leads to the formation of U-loops starting from the sunspot canopy is seen in the simulations of Schlichenmaier (2002). Another possibility is flux pumping by granulation. It has been invoked by Thomas et al. (2002) to pull and keep field lines or flux tubes down below the surface. Here we point out yet another possible mechanism for the formation of U-loops acting on the Evershed flow in sunspot canopies.

The Evershed flow represents a nearly horizontal flow of matter directed radially outward in the penumbra. Shine et al. (1994) and Rimmele (1994) have argued on the basis of filtergram movies that the flow is intermittent. This has recently been confirmed and put on a solid foundation by Rouppe van der Voort (2002) using spectroscopic data. Consider a packet of dense outward flowing gas. Within the penumbra this packet, which cools as it flows along a horizontal flux tube in the penumbra (Schlichenmaier et al. 1999), is prevented from sinking by magnetic forces. The substantial vertical field strength gradient of 1-2 $\mathrm{G} \mathrm{km}^{-1}$ (e.g. Bruls et al. 1995; cf. Solanki 2002) provides a force that resists the gravitational force (note that plasma $\beta<1$ in the penumbra; Solanki et al. 1993). At the edge of the penumbra this supporting force disappears and a sufficiently dense and massive packet of Evershed gas cannot be supported by the flux-tube field any more. This gas then sinks, taking the magnetic field with it. In this way a Uloop is created near the penumbral edge. Submergence of field and a downflow near the penumbral boundary has been observed by Westendorp Plaza et al. (1997), Schlichenmaier \& Schmidt (2000) and Mathew et al. (2002). The forces acting on the U-loop are buoyancy (or rather gravity), which pulls the dense material downwards, and the magnetic tension (curvature force) which pulls the field lines at the bottom of the U upwards.

Which of the proposed mechanisms is relevant for the formation of MMF pairs is not yet clear. A combination of causes is also possible. Irrespective of the exact cause of U-loop formation, the intersections of this U-loop with the solar surface are visible as the two polarities of a bipolar MMF. A sketch of the proposed geometry is given in Fig. 9 (cf. Fig. 8). Note that a U-loop sinking from above represents quite a different situation from a U-loop rising from below, which was studied by Spruit et al. (1987). In the first case the flux tube is relatively evacuated as it reaches the solar surface, in the second case, a lot of material is trapped within it. Hence, in the former case the field strength in the legs of the loop will be enhanced as it sinks and the gas drains down the legs, while in the latter case the field at the solar surface is expected to weaken with time.

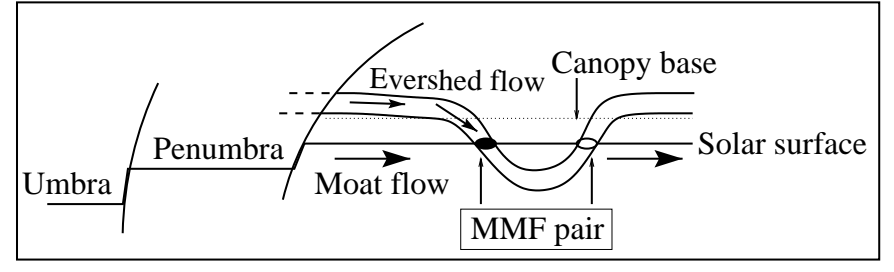

Fig. 9. Sketch showing an MMF pair as the intersection of a U-loop, produced by a downward kink in the field of the superpenumbral canopy, with the solar surface.

Hence, our model in principle allows for kG field strengths in MMFs, which are typical of photospheric flux tubes.

The U-loop emanating from the canopy is driven by the moat flow. Note that the MMF speeds found by us are similar to those of intranetwork magnetic elements (Zhang et al. 1998), which are thought to be dragged along by the supergranular flow. The Evershed flow in the magnetic canopy of $1-2 \mathrm{~km} \mathrm{~s}^{-1}$ (Solanki et al. 1994) is faster, however, than the MMFs, so that fresh material from the sunspot is expected to keep catching up with the MMF. Presumably this material flows down the leg of the MMF and its inertia keeps providing fresh impetus to keep the MMF moving faster than the moat flow. The observation that at least some of the MMFs initially move faster early in their life before slowing down, suggests that the moat flow is the prime driver of older MMFs, but that other mechanisms, e.g. the Evershed flow, act on younger MMFs. It has been shown that less than half of the mass flux seen in the penumbra continues in the magnetic canopy (Solanki et al. 1994, 1999). Our model proposes that the remainder is transported by the MMFs, and that even a part of the flow in the canopy returns to the solar interior within the MMFs. It also suggests that the field lines found to submerge at the edge of the penumbra by Westendorp Plaza et al. (1997) and Mathew et al. (2002) eventually rejoin the magnetic canopy. This is also predicted by the simulations of Schlichenmaier (2002).

According to our U-loop model of MMF pairs, the angle $\alpha$ formed by the axis of an MMF pair with the radial direction to the centre of the sunspot is mainly driven by the direction of the magnetic field in the superpenumbral canopy. However, we expect that the orientation of the U-loops is considerably modified by the granulation flow field that they are embedded in. This could be the cause for the large scatter in $\alpha$ values seen in the top frame of Fig. 7.

The model proposed above is supported by various observations. Firstly, irrespective of the exact cause of U-loop formation the outer arm of a U-loop emanating downwards from the magnetic canopy has the same polarity as the parent sunspot. This agrees with our findings and those of Yurchyshyn et al. (2001). Secondly, a U-loop produced by the submergence of a part of the magnetic canopy naturally explains the observation that MMFs are not visible in the chromospheric He I $10830 \AA$ line (Penn \& Kuhn 1995), but are well seen in brightness diagnostics sampling the upper photosphere (Shine \& Title 2001). Thirdly, the fact that the axes of the MMFs are oriented 
preferentially along the $\mathrm{H} \alpha$ fibrils suggests a connection with the superpenumbral canopy (Yurchyshyn et al. 2001; this paper), as does the alignment of their direction of motion with $\mathrm{H} \alpha$ fibrils. Further support is provided by the fact that the separation between the two polarities of an MMF pair does not change significantly with time (this paper), in contrast to what one would expect for an emerging $\Omega$ loop. However, this observation does not set any strong constraints, since it is also consistent with the hypothesis that the MMFs forming a pair are connected through an O-loop, as proposed by Wilson (1986), but we would expect O-loops rising from below to be randomly oriented and certainly not in the direction of the twisted superpenumbral fibrils (since the white-light sunspots do not exhibit this twist). Also, the fact that more MMF pairs are seen in the direction of the opposite polarity pore/sunspot in AR 8375 can be explained by this model. The presence of a nearby strong, opposite polarity magnetic feature helps to extend the magnetic canopy, and serves as a focus of the field lines. Since in our model the MMF pairs follow the field lines, it follows naturally that they should also be focussed towards the opposite polarity feature (see Figs. 7 and 8). The discovery of U-loop-like structures in the vicinity of a forming sunspot by Bernasconi et al. (2002), although not directly related to MMFs, nevertheless shows that such structures do form in sunspot canopies (see Fig. 9). Finally, the observation that pores are not surrounded by MMFs (Wang et al. 1991) points to the need for a penumbra (and, we would argue, also the need for a superpenumbral canopy) to produce MMFs, again in agreement with our model.

\section{Conclusions}

The large sample of MMF pairs analyzed in this paper allows us to determine their properties in a statistically robust sense. In addition, the use of MDI data, which are free of seeing fluctuations, allows the properties of MMFs to be followed as a function of time without distortions due to the Earth's atmosphere.

The present work confirms the results of Yurchyshyn et al. (2001), as well as revealing further systematics in the properties of MMFs. We argue that these and other observations are best reproduced by a model in which MMFs are the intersections with the solar surface of U-loops produced by localized dips of the magnetic canopy. A sea-serpent-like situation may also be present, so that if one does not restrict oneself to isolated MMF pairs it may, in some cases, be difficult to distinguish between $\mathrm{U}$ - and $\Omega$-loops from the observation. Nonetheless it is difficult to see how the models of Ryntova et al. (1998) and Thomas et al. (2002) for MMF pairs can be reconciled with our results and those of Yurchyshyn et al. (2001).

We cannot rule out that these results apply mainly to relatively young sunspots like the ones we study, while older spots may produce MMFs of another type. This needs to be checked. The investigation of MMFs around a larger sample of sunspots would be a useful exercise, anyway. In addition, it is important to study further properties of MMFs, such as flows within them, their field strengths and field inclinations.
Acknowledgements. We are grateful to V. Martínez Pillet, R. Schlichenmaier and M. Schüssler for helpful discussions. The authors are indebted to the SOHO/MDI and BBSO teams for providing the employed data. SOHO is a mission of international cooperation between ESA and NASA. This work is supported by the National Natural Science Foundation of China under Grant No. G19973009, the National Key Basic Research Science Foundation G2000078404, and the cooperation agreement between the Chinese Academy of Sciences and the Max-Planck Society.

\section{References}

Bernasconi, P. N., Rust, D. M., Georgulis, M. K., \& LaBonta, B. J. 2002, Sol. Phys., 209, 119

Brickhouse, N. S., \& LaBonta, B. J. 1988, Sol. Phys., 115, 43

Bruls, J. H. M. J., Solanki, S. K., Rutten, R. J., \& Carlsson, M. 1995, A\&A, 293, 225

Harvey, K., \& Harvey, J. 1973, Sol. Phys., 28, 61

Holzwarth, V., \& Schüssler, M. 2002, in preparation

Lee, J. W. 1992, Sol. Phys., 139, 267

Martínez Pillet, V. 2002, Astron. Nachr., 323, 342

Mathew, S. K., Solanki, S. K., Lagg, A., et al. 2002, in Poster Proc. 1st Potsdam Thinkship on Sunspot \& Starspots, ed. K. Strassmeier, AIP, 117

Muller, R., \& Mena, B. 1987, Sol. Phys., 112, 295

Penn, M. J., \& Kuhn, J. R. 1995, ApJ, 441, L51

Rimmele, T. R. 1994, A\&A, 290, 972

Rouppe van der Voort, L. 2002, in Poster Proc. 1st Potsdam Thinkship on Sunspot \& Starspots, ed. K. Strassmeier, AIP, 27

Ryutova, M., Shine, R., Title, A., \& Sakai, J. I. 1998, ApJ, 492, 402

Scherrer, P. H., Bogart, R. S., Bush, R. I., et al. 1995, Sol. Phys., 162, 129

Schlichenmaier, R. 2002, Astron. Nachr., 323, 303

Schlichenmaier, R., \& Schmidt, W. 2000, A\&A, 358, 1122

Schlichenmaier, R., Bruls, J. H. M. J., \& Schüssler, M. 1999, A\&A, 349, 961

Shine, R. A., Title, A. M., Tarbell, T. D., et al. 1994, ApJ, 430, 413

Shine, R., \& Title, A. 2001, Encyclopedia of Astron. Astrophys., Vol. 4, 3209

Solanki, S. K. 2002, Astron. Astrophys. Rev., in press

Solanki, S. K., Walther, U., \& Livingston, W. 1993, A\&A, 277, 639

Solanki, S. K., Montavon, C. A. P., \& Livingston, W. 1994, A\&A, 283, 221

Solanki, S. K., Finsterle, W., Rüedi, I., \& Livingston, W. 1999, A\&A, 347, L27

Spruit, H. C., Title, A. M., \& van Ballegooijen, A. A. 1987, Sol. Phys., 110,115

Thomas, J. H., Weiss, N. O., Tobias, S. M., \& Brummell, N. H. 2002, Nature, 420, 390

Vrabec, D. 1971, in ed. R. Howard, Solar Magnetic Fields, IAU Symp., 43, 201

Wang, H., Zirin, H., \& Ai, G. 1991, Sol. Phys., 131, 53

Westendorp Plaza, C., del Toro Iniesta, J. C., Ruiz Cobo, B., et al. 1997, 1st Advances in Solar Physics Euroconference. Advances in Physics of Sunspots, ed. B. Schmieder, J. C. del Toro Iniesta, \& M. Vazquez, ASP Conf. Ser., 118, 202

Wilson, P. R. 1973, Sol. Phys., 32, 435

Wilson, P. R. 1986, Sol. Phys., 106, 1

Yurchyshyn, V. B., Wang, H., \& Goode, P. R. 2001, ApJ, 550, 470

Yurchyshyn, V. B., \& Wang, H. 2001, Sol. Phys., 203, 233

Zhang, J., Wang, J., Wang, H., \& Zirin, H. 1998, A\&A, 335, 341 